\title{
57 Surgery of congenital ear anomalies
}

A The stapedius tendon is absent in $1 \%$ of cases.

B The facial nerve takes an abnormal course in about $30 \%$ of cases and the fallopian canal is dehiscent in $6 \%$.

C A persistent stapedial artery, if present, usually crosses the stapes footplate posteriorly.

D The malleus is the most commonly malformed ossicle.

E An identifiable tragus is usually associated with a partially formed external meatus.

\section{Surgical correction of congenital atresia of the external auditory meatus}

A Cases of unilateral atresia do not require investigation.

B Exploration in bilateral cases can be deferred until about 4 years of age.

C Plastic reconstruction of the auricle is relatively easy.

D Restenosis of a reconstructed external canal is a frequent problem.

E External ear remnants are good indicators of the location of the middle ear.

\section{Traumatic perforation of the tympanic membrane}

A Blast rupture affects the pars flaccida.

B Tinnitus and vertigo are permanent symptoms.

C Blood clot should be syringed out immediately.

D Myringoplasty should be performed as early as possible and always within the first 3 weeks.

E Severe pain, requiring analgesia, continues for several days.

60 In basal skull fractures involving the petrous temporal bone

A Longitudinal fractures are usually caused by blows to the occipital or frontal regions.

B Transverse fractures commonly cause facial paralysis.

C Haemotympanum may present as a blue or black eardrum.

D Ecchymosis over the mastoid area may be present.

E Sensorineural deafness is irreversible. 